\title{
Research on Supply Chain Inventory Optimization and Benefit Coordination with Controllable Lead Time
}

\author{
Fei Ye，Yi-Na Li， Xue-Jun Xu and Jian-Hui Zhao
}

School of Business Administration, South China University of Technology, Guangzhou, P.R.China 510640

\begin{abstract}
In this paper, we propose two supply chain inventory models with controllable lead time, the first is proposed under centralized decision mode and the other is proposed under decentralized decision mode. The solution procedures are also suggested to get the optimal solutions. In addition, taking individual rationality into consideration, Shapely value method and MCRS method are used to coordinate the benefits of the vendor and the buyer. Numerical example is given to illustrate the results of the proposed models.
\end{abstract}

Keywords: Supply Chain Inventory Model, Controllable Lead Time, Shapely Value, MCRS Method

\section{Introduction}

Time-based competition (TBC) has been one of the most popular competitive modes and time management is becoming more and more important in this increasing intense competitive environment [1]. In most of traditional economic order quantity literatures, lead time is viewed as a constant or a stochastic variable by either using deterministic or probabilistic models, that is, lead time is assumed to be not subject to control [2,3]. However, this may not be realistic. As pointed out by Tersine [4], lead time usually is consisted of five components: order preparation time, order transmit time, vendor's lead time, delivery time and setup time. In many practical cases, lead time can be reduced by an added crashing cost, that is, it is controllable. The benefit of reducing lead time, such as lower safety stock, decrease stock out loss, enhance customer service level, and obtain the competitive advantages has been clearly evidenced by successful experience of using Just-In-Time (JIT) production. Lead time reduction has been viewed to be an effective way to realize the quick response of the whole supply chain and one of the most important sources of competitive advantage [1].

Liao \& Shyu (1991) [5] first put forward a continuous review model where order quantity is predetermined and lead time is the only decision variable. Ben-Daya \& Raouf (1994 ) [6] extended Liao \& Shyu’s model (1991) by viewing both lead time and order quantity as decision variables. Ouyang et al. (1996) [7] further took the assumption that the shortage could be divided into a mixture of backorders and lost sales. Ouyang\&Wu (1998) [8] viewed that the demand of lead time can be any known and free cumulative distribution, and proposed procedures to get the optimal lead time and order quantity under different situations. Moon\&Choi (1998) [9] extended Ouyang et al.'s model (1996) by considering the reorder point to be another decision variable. Ouyang\&Chang (2000) [10] improved Ouyang et al.'s model (1996 ) by further assuming the backorder rate is not a determined constant, but be dependent on the length of lead time. All of these above models only took the optimal policy decisions for the buyer into consideration. However, the growing focus on supply chain management for this increasing intense competitive environment calls for a more efficient management of inventories across the whole supply chain through more coordination and cooperation. In recent paper, Pan \& Yang (2002) [11] extended Goyal's model (1988) [12] by assuming lead time as a controllable variable and gained a lower joint expected cost and shorter lead time of the entire supply chain compared to that of Goyal's model. Ouyang et al. (2004) [13] improved Pan \& Yang's model (2002) by further assuming the reorder point as the other decision variable and shortages is permitted, optimizing ordering quantity, lead time, reorder point and the number of lots simultaneously in an integrated supply chain inventory model.

In this paper, we consider the single vendor single buyer inventory problem. As known to all, a supply chain can be viewed as a network which is consisted of series of suppliers, manufacturers, retailers, and customers, through the physical flow, information flow and financial flow. It is beginning with the raw materials producing by a supplier and ending with the product consumption by customers. A node in the supply chain network represents a physical site, a sub-network, or an operation process, and links represent physical flow. However, all supply chain network (SCN) can be divided into several one-to-one supply models consists of single vendor and single buyer under certain conditions. This kind of one-to-one supply model is the basis of supply chain network analysis. Hence, we only take the two-echelon 
supply chain consists of single vendor and single buyer situation into consideration in this paper. We relax the assumption that long-term strategic partnerships between vendor and buyer were well established and they could bargaining and cooperate with each other to obtain an optimal integrated joint policy under centralized decision mode in both Pan \& Yang model (2002) [11] and Ouyang et al. model (2004) [13]. We assume the vendor and the buyer representing different benefit entities and take their individual rationalities into consideration, develop two effective benefit sharing models to coordinate benefit between vendor and buyer and realize the Pareto dominance of the entire supply chain system. Solution procedures are suggested for solving the proposed models and numerical examples are provided to illustrate the results.

This paper is organized as follows. In the section 2, two different inventory models with controllable lead time are proposed, one is proposed under centralized decision mode, and other is proposed under decentralized decision mode. The solution procedures are also suggested to get the optimal solutions. In the section 3, a numerical example is provided to illustrate the results of the proposed models. Shapley value method and MCRS method are used to coordinate the benefits of the vendor and the buyer in section 4 and section 5 contains some concluding remarks and future research.

\section{Model Construction}

\subsection{Notations and Assumptions}

To develop the proposed models, the following notations are used.

$D=$ Average demand per year;

$P=$ Vendor's production rate. $(P>D)$;

$A=$ Buyer's ordering cost per order;

$h_{r}=$ Buyer's unit holding cost per year;

$S=$ Vendor's setup cost per set-up;

$h_{s}=$ Vendor's unit holding cost per year;

$Q=$ Order quantity of the buyer (Decision variable);

$L=$ Length of lead time (Decision variable);

$\gamma=$ Unit shortage cost.

The following assumptions are made for both models in this paper:

1. A two-echelon supply chain consists of single vendor and single buyer is considered.

2. Inventory is continuously reviewed and replenishments are made whenever the inventory level falls to the reorder point $r$.

3. The reorder point $r$ =expected demand during lead time + safety stock. The demand $X$ during lead time $L$ is assumed to be normally distributed with mean $u L$ and standard deviation $\delta \sqrt{L}$. That is, $r=u L+k \delta \sqrt{L}$ where $k$ is the safety factor.

4. The vendor manufactures the product in lots of size $m Q$ with a finite production rate $P(P>D)$ and ship in quantity $Q$ to the buyer over $m$ lots.

5. The lead time has $n$ mutually independent components. The $i$ th component has a minimum duration $a_{i}$ and normal duration $b_{i}$, buyer's crashing cost per unit time $c_{i}$ and vendor's crashing cost per unit time $d_{i}$. Furthermore, for convenience, we arrange $c_{i}$ and $d_{i}$ such that $c_{1} \leq c_{2} \leq \ldots \leq c_{n}$, $d_{1} \leq d_{2} \leq \ldots . \leq d_{n}$. Then, it is clear that the reduction of lead time should be first on component 1 (because it has the minimum unit crashing cost), and then component 2, and so on.

6. If we let $L_{0}=\sum_{j=1}^{n} b_{j}$ and $L_{i}$ be the length of lead time with components $1,2, \ldots . . i$ crashed to their minimum duration, then $L_{i}$ is expressed as

$$
\begin{aligned}
& L_{i}=\sum_{j=1}^{i} a_{j}+\sum_{j=i+1}^{n} b_{j}=\sum_{j=1}^{n} b_{j}-\sum_{j=1}^{i}\left(b_{j}-a_{j}\right)=L_{0}-\sum_{j=1}^{i}\left(b_{j}-a_{j}\right) \\
& i=1,2, \ldots . n .
\end{aligned}
$$

\subsection{Buyer's inventory cost model}

Based on the above notations and assumptions, the total expected annual cost for the buyer is given by:

\section{$T E C_{r}=$ ordering cost + holding cost + lead time crashing cost + shortage cost}

Since $A$ is the ordering cost per order, the expected ordering cost per year is given by $D A / Q$.

The average on-hand inventory for the buyer is $I_{r}=\frac{Q}{2}+k \delta \sqrt{L}$ and the expected holding cost per year for the buyer is $h_{r}\left(\frac{Q}{2}+k \delta \sqrt{L}\right)$.

The buyer's lead time crashing cost $R(L)$ for a given $L \in\left[L_{i}, L_{i-1}\right]$ is given by

$R(L)=c_{i}\left(L_{i-1}-L\right)+\sum_{j=1}^{i-1} c_{j}\left(b_{j}-a_{j}\right)$, hence the expected annual lead time crashing cost for the buyer is $D R(L) / Q$.

The expected shortage of each order cycle is $E(X-r)^{+}=\int_{R O P}^{+\infty}(X-r) d F(x)=\delta \sqrt{L} \Psi(k)$, where $\Psi(k)=\phi(k)-k[1-\Phi(k)]$, and $\phi, \Phi$ are the standard normal distribution and cumulative distribution function, respectively [14]. The expected shortage cost per year is $D \gamma \delta \sqrt{L} \Psi(k) / Q$.

Therefore, the total expected annual cost for buyer is given by 
$T E C_{r}(Q, L)=\frac{D A}{Q}+h_{r}\left(\frac{Q}{2}+k \delta \sqrt{L}\right)+\frac{D}{Q} R(L)+\frac{D \gamma}{Q} \delta \sqrt{L} \Psi(k)$

\subsection{Vendor's inventory cost model}

For the vendor's inventory model, its total expected annual cost can be represented by:

\section{$T E C_{v}=$ set-up cost + holding cost + lead time crashing cost}

Since $S$ is the vendor's set-up cost per set-up, and the production quantity in a lot will be $Q$, the expected set-up cost per year is given by $D S / Q$.

The vendor's average inventory can be evaluated as $Q D / 2 P$. Hence, the vendor's expected holding cost per year is $h_{s} Q D / 2 P$.

The vendor's lead time crashing cost $M(L)$ for a given $L \in\left[L_{i}, L_{i-1}\right]$ is given by

$M(L)=d_{i}\left(L_{i-1}-L\right)+\sum_{j=1}^{i-1} d_{j}\left(b_{j}-a_{j}\right)$, hence the expected annual lead time crashing cost for the vendor is $D M(L) / Q$.

It follows that the total expected annual cost for the vendor is:

$T E C_{s}=\frac{D}{Q} S+h_{s} \frac{Q}{2}\left(\frac{D}{P}\right)+\frac{D}{Q} M(L)$

\subsection{Inventory model under centralized mode}

To provide a benchmark, we first analyze the supply chain system where a central controller makes all decisions to minimize the total expected cost of the whole supply chain. In this case, the vendor and the buyer negotiate to decide lead time and order quantity together. The integrated inventory of supply chain under centralized mode is given by

$$
\begin{aligned}
& \text { TEC }_{s c}(Q, L)=\frac{D}{Q}(A+S+R(L)+M(L))+h_{r}\left(\frac{Q}{2}+k \delta \sqrt{L}\right) \\
& +\frac{D \gamma}{Q} \delta \sqrt{L} \Psi(k)+h_{s} \frac{Q}{2}\left(\frac{D}{P}\right)
\end{aligned}
$$

Taking the partial derivatives of $T E C_{s c}(Q, L)$ with respect to $Q, L$ in each time interval $\left[L_{i}, L_{i-1}\right]$, and equating them to zero, we obtain

$$
\begin{aligned}
& \frac{\partial \operatorname{TEC}_{s c}(Q, L)}{\partial Q}=-\frac{D}{Q^{2}}(A+S+R(L)+\gamma \delta \sqrt{L} \Psi(k)+M(L)) \\
& +\frac{h_{r}}{2}+\frac{h_{s} D}{2 P}=0
\end{aligned}
$$

$\frac{\partial T E C_{s c}(Q, L)}{\partial L}=\frac{D}{Q}\left(\frac{\gamma \delta L^{-\frac{1}{2}} \Psi(k)}{2}-c_{i}-d_{i}\right)+\frac{h_{r} k \delta L^{-\frac{1}{2}}}{2}=0$

Hence, for fixed $L \in\left[L_{i}, L_{i-1}\right], \quad T E C_{s c}(Q, L)$ is convex in $Q$, since

$\frac{\partial T E C_{s c}(Q, L)}{\partial Q^{2}}=\frac{2 D}{Q^{3}}(A+S+R(L)+M(L)+\gamma \delta \sqrt{L} \Psi(k))>0$

However, for fixed $Q, T E C_{s c}(Q, L)$ is concave in $L \in\left[L_{i}, L_{i-1}\right]$, because

$$
\frac{\partial T E C_{s c}(Q, L)}{\partial L^{2}}=-\frac{D}{Q} \frac{\gamma \delta L^{-\frac{3}{2}} \Psi(k)}{4}-\frac{h_{r} k \delta L^{-\frac{3}{2}}}{4}<0
$$

Therefore, for fixed $Q$, the minimum expected annual cost of the entire supply chain will occur at the end points of the interval $\left[L_{i}, L_{i-1}\right]$. From Eq. (4), we have

$$
Q^{*}=\sqrt{\frac{2 P D(A+S+R(L)+M(L)+\gamma \delta \sqrt{L} \Psi(k))}{h_{r} P+h_{s} D}}
$$

We have proved that the total expected annual cost $T E C_{s c}(Q, L)$ is convex in $Q$ and easily obtain the analytic expression of the optimal order quantity under centralized mode. However, we assume the lead time crashing cost to be a piecewise linear function and have proved that the total expected annual cost $\operatorname{TEC}_{s c}(Q, L)$ is concave in $L \in\left[L_{i}, L_{i-1}\right]$ and the minimum $T E C_{s c}(Q, L)$ will occur at the end points of the interval $\left[L_{i}, L_{i-1}\right]$. So we cannot obtain the analytic expression of the optimal lead time directly. Hence we can develop the following heuristic algorithm 1 to get the optimal values of $Q, L$ under centralized mode. We can compare the total expected annual cost of each end point of $\left[L_{i}, L_{i-1}\right]$ and set the lead time and order quantity that minimizing total expected annual cost to be the optimal lead time and order quantity decisions.

\section{Algorithm 1}

Step1: For each $L_{i},(i=0,1, \ldots, n)$, compute $Q_{i}$ using Eq. (8).

Step2: For each $\left(L_{i}, Q_{i}\right)$, compute the expected annual cost of the entire supply chain $T E C_{s c}\left(Q_{i}, L_{i}\right)$, $i=0,1,2, \ldots, n$.

Step3: $\operatorname{Set} T E C_{s c}\left(Q^{*}, L^{*}\right)=\min _{i=0,1,2, ., n} T E C_{s c}\left(Q_{i}, L_{i}\right)$, then $\left(Q^{*}, L^{*}\right)$ is a set of optimal solutions under centralized mode.

\subsection{Inventory model under decentralized mode}


Under decentralized mode, the buyer and the vendor do not cooperate with each other, they will determine their own optimal policy separately. That is, the buyer will choose optimal order quantity and lead time to maximum his own benefit. Hence, taking the partial derivatives of $T E C_{r}(Q, L)$ in Eq. (1) with respect to $Q$ and $L$ in each time interval $\left[L_{i}, L_{i-1}\right]$, and equating them to zero, we obtain

$$
\begin{aligned}
& \frac{\partial T E C_{r}(Q, L)}{\partial Q}=-\frac{D}{Q^{2}}(A+R(L)+\gamma \delta \sqrt{L} \Psi(k))+\frac{h_{r}}{2}=0 \\
& \frac{\partial T E C_{r}(Q, L)}{\partial L}=\frac{D}{Q}\left(\frac{\gamma \delta L^{-\frac{1}{2}} \Psi(k)}{2}-c_{i}\right)+\frac{h_{r} k \delta L^{-\frac{1}{2}}}{2}=0
\end{aligned}
$$

Hence, for fixed $L \in\left[L_{i}, L_{i-1}\right], T E C_{r}(Q, L)$ is convex in $Q$, since

$$
\frac{\partial T E C_{r}^{2}(Q, L)}{\partial Q^{2}}=\frac{2 D}{Q^{3}}(A+R(L)+\gamma \delta \sqrt{L} \Psi(k))>0
$$

However, for fixed $Q, \quad \operatorname{TEC}_{r}(Q, L)$ is concave in $L \in\left[L_{i}, L_{i-1}\right]$, because

$$
\frac{\partial T E C_{r}^{2}(Q, L)}{\partial L^{2}}=-\frac{\gamma \delta L^{-\frac{3}{2}} \Psi(k) D}{4 Q}-\frac{h_{r} k \delta L^{-\frac{3}{2}}}{4}<0
$$

Therefore, for fixed $Q$, the buyer's minimum expected annual cost will occur at the end points of the inter$\operatorname{val}\left[L_{i}, L_{i-1}\right]$. From Eq. (9), we have

$$
Q^{* * *}=\sqrt{\frac{2 D(A+R(L)+\gamma \delta \sqrt{L} \Psi(k))}{h_{r}}}
$$

In the same way of the situation of centralized mode, we proved that the buyer's expected annual cost $T E C_{r}(Q, L)$ is convex in $Q$ and easily obtain the analytic expression of the optimal order quantity under decentralized mode. However, we assume the lead time crashing cost to be a piecewise linear function and proved that the buyer's expected annual cost $\operatorname{TEC}_{r}(Q, L)$ is concave in $L \in\left[L_{i}, L_{i-1}\right]$ and the minimum $\operatorname{TEC}_{r}(Q, L)$ will occur at the end points of the interval $\left[L_{i}, L_{i-1}\right]$. So we cannot obtain the analytic expression of the optimal lead time directly. Hence we can develop the following heuristic algorithm 2 similar to algorithm 1 to get the optimal values of $Q, L$ under decentralized mode.

\section{Algorithm 2}

Step1: For each $L_{i},(i=0,1, \ldots, n)$, compute $Q_{i}$ using Eq. (13).
Step2: For each $\left(L_{i}, Q_{i}\right)$, compute the buyer's expected annual cost $T E C_{r}\left(Q_{i}, L_{i}\right), i=0,1,2, \ldots, n$.

Step3: $\operatorname{Set} T E C_{r}\left(Q^{* *}, L^{* *}\right)=\min _{i=0,1,2, ., n} T E C_{r}\left(Q_{i}, L_{i}\right)$, then $\left(Q^{* *}, L^{* *}\right)$ is a set of optimal solutions under decentralized mode. And the vendor's and buyer's expected cost under decentralized mode is $T E C{ }_{s}\left(Q^{* *}, L^{* *}\right), T E C_{r}\left(Q^{* *}, L^{* *}\right)$, respectively.

\section{Numerical Example}

Consider an inventory system with the following characteristics: $D=600$ unit/year , $P=2500$ unit / year , $h_{r}=\$ 20 /$ unit / year , $A=\$ 200 /$ order,$\delta=7$ unit $/$ week , $\gamma=\$ 60 /$ unit,$h_{s}=\$ 40 /$ unit / year,$S=\$ 250 /$ set - up , $k=2$. The lead time has three components with the data shown in Table 1.

Table 1. Lead time data (i: Component of lead time; $a_{i}$ : Minimum duration with crashing; $b_{i}$ Normal duration; $c_{i}$ : Buyer's crashing cost per unit time; $d_{i}$ : Vendor's crashing cost per unit time)

\begin{tabular}{ccccc}
\hline$i$ & $b_{i}$ (days) & $a_{i}$ (days) & $c_{i}(\$ /$ day $)$ & $d_{i}(\$ /$ day $)$ \\
\hline 1 & 20 & 6 & 0.4 & 0 \\
2 & 20 & 6 & 1.2 & 2.0 \\
3 & 16 & 9 & 5.0 & 3.0 \\
\hline
\end{tabular}

The results under the centralized decision mode are summarized in Table 2 and the results under the decentralized decision mode are summarized in Table 3.

\begin{tabular}{|c|c|c|c|c|c|c|c|}
\hline \multirow{2}{*}{$i$} & \multirow{2}{*}{$L$} & \multirow{2}{*}{$R\left(L_{i}\right)$} & \multirow{2}{*}{$M\left(L_{i}\right)$} & \multirow{2}{*}{$Q_{i}$} & \multirow{2}{*}{$x$} & \multicolumn{2}{|c|}{$y$} \\
\hline & & & & & & $y_{1}$ & $y_{2}$ \\
\hline 0 & 8 & 0 & 0 & 136 & 4832 & 1754 & 3078 \\
\hline 1 & 6 & 5.6 & 0 & 137 & $4745^{*}$ & 1752 & 2993 \\
\hline 2 & 4 & 22.4 & 28 & 139 & 4804 & 1851 & 2953 \\
\hline 3 & 3 & 57.4 & 49 & 144 & 4954 & 1913 & 3041 \\
\hline
\end{tabular}

Table 2. Summary of the results under centralized decision mode ( $x$ : Expected cost of supply chain; $y$ : Both parties' expected cost without coordination; $y_{1}$ : Vendor; $y_{2}$ : Buyer)

Table 3. Summary of the results under decentralized decision mode ( $r$ : Inventory cost of supply chain; $s$ : Vendor's expected cost; $t$ : Buyer's expected cost)

\begin{tabular}{ccccccc}
\hline$i$ & $L_{i}$ & $R\left(L_{i}\right)$ & $Q_{i}$ & $r$ & $s$ & $t$ \\
\hline 0 & 8 & 0 & 112 & 4910 & 1876 & 3034 \\
1 & 6 & 5.6 & 113 & 4819 & 1868 & 2951 \\
2 & 4 & 22.4 & 117 & 4890 & 1985 & $2905^{*}$ \\
3 & 3 & 57.4 & 126 & 5029 & 2031 & 2998 \\
\hline
\end{tabular}

From Table 2, the optimal inventory policy under cen- 
tralized mode can be easily obtained. The optimal lead time $L^{*}=6$ weeks, optimal order quantity $Q^{*}=137$ units . The minimum expected annual cost of the entire supply chain is $T E C_{s c}=\$ 4745$, and the vendor's and buyer's expected costs are \$1752 and \$2993, respectively.

From Table 3, the optimal inventory policy under decentralized mode can be easily obtained. The optimal lead time $L^{* *}=4$ weeks, optimal order quantity $Q^{* *}=117$ units . The buyer's minimum expected cost is \$2905, and the vendor's expected cost is $\$ 1985$, then the inventory cost of the entire supply chain is $\$ 4890$.

Obviously, the expected annual cost of the entire supply chain under decentralized decision mode is higher than that of centralized decision mode. However, the buyer's expected cost under centralized mode is higher than that of decentralized mode. Hence, taking individual rationality into consideration, we need to design mechanisms that can induce both the vendor and the buyer to cooperate and make decisions to minimum the expected annual cost of the entire supply chain. In the following, we develop two kinds of benefit sharing methods, the first one is based on Shapley value method and the other is based on MCRS method (Minimum Costs-Remaining Savings) to coordinate the benefits of the vendor and the buyer. These two benefit sharing methods can not only meet both vendor and buyer's individual rationality, but also realize Pareto dominance of the entire supply chain.

\section{Supply Chain Benefit Allocation Model}

The benefit allocation methods to make benefit sharing of multiple-person cooperation game usually include Shapely value method, core method, CGA (Cost Gap Allocation) method and MCRS (Minimum Costs-Remaining Savings) method. Here we adopt Shapley value method and MCRS method to allocate benefits of supply chain.

\subsection{Shapley value method}

The Shapley value is one of the most popular benefit allocation solutions for the cooperative games. By using Shapley value method, we can suggest an allocation criterion to the benefits obtained from the cooperation among the players who have cooperated to form a coalition [15]. The Shapley value method gave out a formula for providing a standard to measure the contribution of each player makes to the benefits of a cartel within a cooperative game [16].

Assuming the number of players taking part into a coalition to be specified and is denoted by $n$. The marginal savings which each player contributes to a coalition depends on the size of that coalition. Let $|T|$ represents the set of players in a coalition before player $i$ 's joining. The saving derived from the inclusion of the $i$ th player in a coalition of size $|N|$ has been given out by the Shapley value method.

For given cooperative game $v$ of $n$ person, there exists single Shapley value $\varphi(v)=\left(\varphi_{1}(v), \varphi_{2}(v), \ldots, \varphi_{n}(v)\right)$, and

$\varphi_{i}[v]=\sum_{T \subseteq N, i \in T} \frac{(|T|-1) !(n-|T|) !}{n !} \cdot[v(T)-v(T \backslash i)], \forall i \in N$

where $v$ is the characteristic function defined in the subset of $N,|T|$ represents numbers of element in coalition $T$, and $n=|N| \cdot v(T \backslash i)$ is the savings of player $i$ joining the coalition of $T, v(T)$ is the savings of the coalition of $T$.

For the case of this paper, only one coalition is possible, for there is only one buyer and one vendor. The benefit of buyer and vendor under decentralized mode is $-T E C_{r}\left(Q^{* *}, L^{* *}\right),-T E C_{s}\left(Q^{* *}, L^{* *}\right)$ respectively. The benefit of the entire supply chain under centralized mode is $-T E C_{s c}\left(Q^{*}, L^{*}\right)$. According to Shapley value we can get the benefit of vendor and buyer under centralized mode, that is

$$
\varphi_{s}[v]=\frac{\left(-T E C_{s c}\left(Q^{*}, L^{*}\right)+T E C_{r}\left(Q^{* *}, L^{* *}\right)\right)+\left(-T E C_{s}\left(Q^{* *}, L^{* *}\right)\right.}{2}
$$

$$
\varphi_{r}[v]=\frac{\left(-T E C_{S C}\left(Q^{*}, L^{*}\right)+T E C_{S}\left(Q^{* *}, L^{* *}\right)\right)+\left(-T E C_{r}\left(Q^{* *}, L^{* *}\right)\right.}{2}
$$

Now we use Eq. (14) and (15) to coordinate the benefit of the vendor and the buyer of this example. Under decentralized decision mode, the expected costs of the buyer and the vendor are $\operatorname{TEC}_{r}\left(Q^{* *}, L^{* *}\right)=\$ 2905$, $T E C_{s}\left(Q^{* *}, L^{* *}\right)=\$ 1985$, respectively. Under centralized decision mode, the expected annual cost of the entire supply chain is $T E C_{s c}\left(Q^{*}, L^{*}\right)=\$ 4745$. So by using Eq. (14) and (15), we can get the benefit of the vendor and the buyer under centralized decision mode, they are $\varphi_{s}[v]=-\$ 1913, \varphi_{r}[v]=-\$ 2832$, respectively. That is, under centralized mode and with benefit coordination, the vendor's expected cost will be $\$ 1913$ and the buyer's expected cost will be $\$ 2832$. From table 2, we can see, under centralized mode and without benefit coordination, the vendor's expected cost is \$1752 and the buyer's expected cost is $\$ 2993$. That is, only if the vendor transfers $\$ 1913-\$ 1752=\$ 161$ to the buyer, and the buyer's expected cost changes to $\$ 2993-\$ 161=\$ 2832$. Then both the vendor's and the buyer's cost will be improved comparing to that of decentralized mode and they will cooperate and make decisions under centralized mode to minimize the expected annual cost of the entire supply chain. Hence the benefit allocation model based on Shapley value method can not only meet both vendor and buyer's individual rationality, but also realize Pareto dominance of 
the entire supply chain.

\subsection{MCRS method}

MCRS method (Minimum Costs-Remaining Savings) is another kind of acknowledged method to allocation benefit of multiple-person cooperation game. It can also be used to coordinate the benefit of each player of supply chain.

Taking the numerical example of this paper for example, we set $T E C_{r}{ }^{*}, T E C_{s}^{*}$ to be the expected annual cost of the buyer and the vendor under centralized decision mode, respectively. According to allocation model of MCRS method, the expected cost of players is given by:

$$
\begin{aligned}
& T E C_{k}^{*}=T E C_{k \min }+ \\
& \frac{T E C_{k \max }-T E C_{k \min }}{\sum_{k=s, r}\left(T E C_{k \max }-T E C_{k \min }\right)}\left[T E C_{s c}\left(Q^{*}, L^{*}\right)-\sum_{k=s, r} T E C_{k \min }\right]
\end{aligned}
$$

where $k=s, r$. TEC $C_{k m i n}, T E C_{k \text { min }}$ can be obtained by linear programming:

$$
\max \text { or } \min T E C_{k}^{*}
$$

$$
\text { s.t. }\left\{\begin{array}{l}
\operatorname{TEC}_{r}\left(Q^{* *}, L^{* *}\right) \geq T E C_{k}^{*} \\
\operatorname{TEC}_{s}\left(Q^{* *}, L^{* *}\right) \geq T E C_{k}^{*} \\
T E C_{k}^{*}+T E C_{k}^{*}=T E C_{s c}\left(Q^{*}, L^{*}\right)
\end{array}\right.
$$

From table 2 and table 3, We obtain $T E C_{r}\left(Q^{* *}, L^{* *}\right)=\$ 2905, \quad T E C_{s}\left(Q^{* *}, L^{* *}\right)=\$ 1985$, $T E C_{s c}\left(Q^{*}, L^{*}\right)=\$ 4745$. By Eq. (13) we can get the optimal solution $T E C_{r \max }{ }^{*}=\$ 2905, T E C_{r \min }{ }^{*}=\$ 2759$, and $T E C_{\text {min }}{ }^{*}=\$ 1840, T_{E C}{ }^{*}{ }^{*}=\$ 1985$. Hence using Eq.(17) we can get allocation solution :

$$
\begin{aligned}
& \text { TEC }_{r}^{*}=2759+\frac{(2905-2759)}{(2905-2759)+(1985-1840)} *[4745-4599] \\
& =\$ 2832
\end{aligned}
$$

$$
\begin{aligned}
& \text { TEC }_{s}^{*}=1840+\frac{(2905-2759)}{(2905-2759)+(1985-1840)} *[4745-4599] \\
& =\$ 1913
\end{aligned}
$$

Hence, we can see the results are consistent with that of Shapley value. MCRS method can also make reasonable allocation of benefits derived from the cooperation between the vendor and the buyer according to their contribution to the coalition.

\section{Conclusions}

In this increasing intense competitive world, more and more companies have recognized the importance of the response time to customer and also have used time man- agement as an important mean of gaining competitive advantage in the global marketplace. Lead time is an important element in any inventory system. In many practical situations, lead time can be controllable by an added crashing cost. In this paper, the supply chain inventory optimization with controllable lead time under centralized mode and decentralized mode are proposed. The solution procedures to get the optimal solutions are also suggested. At last, the benefit allocation models based on Shapley value method and MCRS method are developed to coordinate the benefit of the vendor and the buyer. The results of numerical example show that shortening lead time reasonably can reduce inventory cost and the benefit allocation models developed in this paper are effective. In real situations, the supply chain network is more complex than that of two-echelon supply chain consists of single vendor and single buyer discussed in this paper. When we take all the players of the supply chain network into consideration, things will be more complicated and the results may be different. Furthermore, only the benefit allocation of one-to-one problem is discussed in this paper. When we extend the problem to the entire supply chain network, there will be existed many more complex relationships, such as one-to-multi, multi-to-multi relationships and so on. How to deal with the cooperation and benefit allocation of $n$ persons with individual difference and competition under these circumstances will be the points of further research. The supply chain inventory optimization problem with controllable lead time under fuzzy circumstance and asymmetric information situation can be the points of further research.

\section{Acknowledgement}

We are extremely grateful to the anonymous referees for their most insightful and constructive comments and valuable editorial efforts, which have enabled us to improve the manuscript significantly. This research was supported by Guangdong Social Science Foundation (06003), Guangdong Natural Science Foundation (05006576) and SRP project supported by South China University of Technology.

\section{REFERENCES}

[1]. R.J. Tersine, and E.A. Hummingbird, "Lead-time Reduction: The Search for Competitive Advantage”, International Journal of Operations and Production Management, 1995,15(2), pp.8-18.

[2]. E. Naddor, Inventory Systems, Wiley, New York, 1966.

[3]. E.A. Silver, and R. Peterson, Decision Systems for Inventory management and Production Planning, Wiley, New York, 1985.

[4]. R.J. Tersine, Principles of Inventory and materials management. North-Holland, New York, 1982.

[5]. C.J. Liao, and C.H. Shyu, “An Analytical Determi- 
nation of Lead Time with Normal Demand”, International Journal of Operations and Production Management, 1991, 11(9), pp.72-78.

[6]. M. Ben-Daya, and A. Raouf, "Inventory Models Involving Lead Time as Decision Variable”, Journal of the Operational Research Society, 1994, 45, pp.579-582.

[7]. L.Y. Ouyang, N.C. Yen, and K.S. Wu, "Mixture Inventory Model with Backorders and Lost Sales for Variable Lead Time”, Journal of the Operational Research Society, 1996, 47, pp.829-832.

[8]. L.Y. Ouyang, and K.S. Wu, "A Minimax Distribution Free Procedure for Mixed Inventory Model with Variable Lead Time”, International Journal of Production Economics, 1998, 56, pp.511-516.

[9]. I. Moon, and S. Choi, "A Note on Lead Time and Distributional Assumptions in Continuous Review Inventory Models", Computers \& Operations Research, 1998,25, pp.1007-1012.

[10]. L.Y. Ouyang, and B.R. Chuang, "Stochastic Inventory Model Involving Variable Lead Time with a Service Level”, Yugoslav Journal of Operations Research, 2000, 10(1), pp.81-98.
[11]. J.C. Pan, Y.C. Hsiao, and C.J. Lee, "Inventory Model with Fixed and Variable Lead Time Crash Costs Considerations”, Journal of the Operational Research Society, 2002, 53, pp.1048-1053.

[12]. S.K. Goyal, "A Joint Economic-lot-size Model for Purchaser and Vendor: A Comment", Decision Science, 1998, 19, pp.236-241.

[13]. L.Y. Ouyang, K.S. Wu, and C.H. Ho, "Integrated Vendor-Buyer Cooperative Models with Stochastic Demand in Controllable Lead Time”, International Journal of Production Economics, 2004, 92, pp.255-266.

[14]. A. Ravindran, D.T. Phillips, and J.J. Solberg, Operations Research: Principle and Practices, Wiley, New York, 1987.

[15]. D. Perez-Castrillo, and D. Wettstein, "Bidding for the surplus: a non-cooperative approach to the Shapley value", Journal of Economics Theory, 2001,100, pp.274-294.

[16]. J. Quigley, and L. Walls, “Trading reliability targets within a supply chain using Shapley's value”, Reliability Engineering \& System safety, 2007, 92(10), pp.1448-1457.

\section{AUTHORS' BIOGRAPHIES}

Ye Fei: Ph.D. Associate professor, school of business administration, South China University of Technology. Research field: supply chain management, virtual enterprise, Multiple Attribute Decision Makings.

Li Yina: Ph.D. school of business administration, South China University of Technology. Research field: supply chain management.

Xu Xuejun: Ph.D. Professor, school of business administration, South China University of Technology. Research field: Industrial Engineering, Operation Strategy, Supply Chain Management and, etc.

Zhao Jianhui: Student of the school of mathematical science, South China University of Technology. 
